\title{
The Problem of General Relativity for an Orthotropic Solid Sphere
}

\author{
Valery Vasiliev $^{1} \&$ Leonid Fedorov $^{1}$ \\ Institute of Problems of Mechanics, Russian Academy of Sciences, 101 Vernadskogo, Moscow, Russia \\ Correspondence: Valery Vasiliev, Institute of Problems of Mechanics, Russian Academy of Sciences, 101 \\ Vernadskogo, Moscow 119526, Russia. Tel: 7-495-382-2040. E-mail: vvvas@dol.ru
}

Received: October 10, 2012 Accepted: November 6, 2012 Online Published: January 16, 2013

doi:10.5539/apr.v5n1p115

URL: http://dx.doi.org/10.5539/apr.v5n1p115

\begin{abstract}
The paper is concerned with analysis and solution of the static problem of the General Theory of Relativity for an orthotropic solid sphere and the surrounding space. It is shown that, in contrast to liquid and isotropic elastic spherical solids, the singularity does not appear at the center of the sphere whose radius is reduced to the gravitational radius.
\end{abstract}

Keywords: general relativity, spherically symmetric problem, orthotropic solid

\section{Introduction}

The spherically symmetric static problem of the General Theory of Relativity (GTR) was solved by Schwarzschild in 1916 just after the theory was developed. The solution for the empty space (Schwarzschild, 1916a) surrounding the sphere with radius $R$ shows that the radial space metric coefficient becomes singular if the sphere radius approaches the gravitational radius $r_{g}$. However, the analysis of this solution (Synger, 1960) allows us to conclude that the surface with the radius $r_{g}$ is located inside the sphere, and the singularity does not exist in the external space. The solution for inside space simulated with a perfect incompressible liquid (Schwarzschild, 1916b) becomes singular at the sphere center if $R$ approaches $r_{g}$, and a natural question arises as to whether this singularity is actual (Hawking \& Penrose, 1970) or it is a formal result having no physical meaning (Fock, 1959; Logunov \& Mestvirishvili, 1985). It is also can be supposed that the singularity appears in solution for the incompressible liquid only and can disappear for a more realistic material model allowing for stresses and strains induced by the gravitation. Two main problems arise in connection with this question. First, for the sphere material which is more general than a perfect incompressible liquid, the GTR equations are not complete and must be supplemented with the corresponding material constitutive equations (Feynman, Morinigo \& Wagner, 1995). Second, only numerical solutions are possible for a complete set of equations (in case such a set is obtained), but such solutions cannot be used to identify singularities because they do not converge not only for singular problems, but also if the solution of the boundary problem does not exist. Numerical solutions which do not converge at the sphere center have been constructed by Vasiliev and Fedorov (2012) for compressible liquid and elastic solid spheres.

In the present paper, the GTR problem for the orthotropic sphere is studied. As follows from the Theory of Elasticity, singularity does not appear at the center of the orthotropic sphere under some conditions imposed on the material elastic constants. Thus, the singularity at the sphere center can be eliminated, whereas, in the vicinity of the outer surface, the sphere material can be simulated with incompressible liquid and the analytical solution for the incompressible can be used to identify the possible singularity.

\section{Governing Equations}

Consider a spherical solid with radius $R$ surrounded by the infinite empty space. In accordance with the classical Schwarzschild solution (Synge, 1960), the line element in spherical coordinates $r, \theta, \varphi$ is taken as

$$
d s^{2}=g d r^{2}+r^{2}\left(d \theta^{2}+\sin ^{2} \varphi d \varphi^{2}\right)-h d t^{2}
$$

where $g(r)$ and $h(r)$ are the coefficients of the metric tensor of semi-Riemannian space induced by gravitation. For the external $(r \geq R)$ and internal $(0 \leq r \leq R)$ spaces the following conservation equation must be satisfied (Synge, 1960): 


$$
\left(T_{1}^{1}\right)^{\prime}-\frac{2}{r}\left(T_{2}^{2}-T_{1}^{1}\right)+\frac{h^{\prime}}{2 h}\left(T_{1}^{1}-T_{4}^{4}\right)=0
$$

Here, $(\ldots)^{\prime}=d(\ldots) / d r$ and $T_{j}^{i}$ are the components of the energy tensor which for the static problem in spherical coordinates are expressed in terms of the radial, $\sigma_{r}$, and the circumferential, $\sigma_{\theta}$, stresses induced by the gravitation inside of the solid sphere with constant density $\rho$, i.e.,

$$
T_{1}^{l}=\sigma_{r}, T_{2}^{2}=T_{3}^{3}=\sigma_{\theta}, T_{4}^{4}=\rho c^{2}
$$

According to GTR, Equation (2) is identically satisfied if the components of the energy tensor $T_{j}^{i}$ are expressed in terms of the components of the Einstein tensor $G_{j}^{i}$ as

$$
\begin{gathered}
\chi T_{1}^{l}=G_{1}^{l}=-\frac{1}{g}\left(\frac{h^{\prime}}{r h}+\frac{1}{r^{2}}\right)+\frac{1}{r^{2}} \\
\chi T_{2}^{2}=\chi T_{3}^{3}=G_{2}^{2}=-\frac{1}{2 g}\left[\frac{h^{\prime \prime}}{h}-\frac{1}{2}\left(\frac{h^{\prime}}{h}\right)^{2}+\frac{1}{r}\left(\frac{h^{\prime}}{h}-\frac{g^{\prime}}{g}\right)-\frac{g^{\prime} h^{\prime}}{2 g h}\right] \\
\chi T_{4}^{4}=G_{4}^{4}=-\frac{1}{g}\left(\frac{1}{r^{2}}-\frac{g^{\prime}}{r g}\right)+\frac{1}{r^{2}}
\end{gathered}
$$

in which

$$
\chi=8 \pi \gamma / c^{4}
$$

and $\gamma$ is the gravitation constant. Because substitution of Equations (4)-(6) identically satisfies Equation (2), only three of four Equations (2) and (4)-(6) are mutually independent. The simplest set of equations which is used further for analysis includes Equations (2), (4) and (6), whereas Equation (5) is satisfied identically (Synge, 1960). Using Equations (3), we can present the final set of equations in the following form:

$$
\begin{gathered}
\sigma_{r}^{\prime}-\frac{2}{r}\left(\sigma_{\theta}-\sigma_{r}\right)+\frac{h^{\prime}}{2 h}\left(\sigma_{r}-\rho c^{2}\right)=0 \\
\chi \sigma_{r}=-\frac{1}{r g}\left(\frac{h^{\prime}}{h}+\frac{1}{r}\right)+\frac{1}{r^{2}} \\
\chi \rho c^{2}=\frac{1}{r^{2}}\left(r-\frac{r}{g}\right)^{\prime}
\end{gathered}
$$

Consider the solution of these equations for the external and internal spaces.

\section{External Space}

The external space $(r \geq R)$ is empty, so that $\sigma_{r}=\sigma_{\theta}=0$ and $\rho=0$. In this case, Equation (8) is satisfied identically, whereas Equations (9) and (10) reduce to

$$
\frac{h_{e}^{\prime}}{h_{e}}=\frac{1}{r}(g-1),\left(r-\frac{r}{g_{e}}\right)^{\prime}=0
$$

Subscript " $e$ " corresponds to the external space. Integration of Equations (11) yields

$$
g_{e}=\frac{1}{1+C_{1} / r}, h_{e}=C_{2}\left(1+C_{1} / r\right)
$$

This result corresponds to the well known Schwarzschild solution. Determine the integration constants $C_{l}$ and $C_{2}$. At an infinite distance from the sphere, i.e., at $r \rightarrow \infty$, Equations (12) must reduce to the solution of the classical gravitation theory (Landau \& Lifshitz, 1962 ), i.e., to 


$$
g_{e}^{0}=1+2 f / c^{2} h_{e}^{0}=1
$$

where $f=-\gamma m / r$ is Newtonian gravitation potential in which $m$ is the sphere mass. Introduce the so-called gravitational radius

$$
r_{g}=2 m \gamma / c^{2}
$$

Then, the conditions $g_{e}(r \rightarrow \infty)=g_{e}^{0}$ and $h_{e}(r \rightarrow \infty)=h_{e}^{0}$ yield $C_{1}=-r_{g}, \quad C_{2}=1$ and Equations (12) become

$$
g_{e}=\frac{1}{1-r_{g} / r}, h_{e}=1-r_{g} / r
$$

For the Euclidean space, $r_{g}=0$ and $g_{e}=h_{e}=1$.

\section{Geometry of Internal Space}

For the spherical solid, $\rho=$ constant and integration of Equation (10) results in the following expression:

$$
g_{i}=\frac{1}{1-\frac{\chi}{3} \rho c^{2} r^{2}+\frac{C_{3}}{r}}
$$

where subscript " $i$ " corresponds to the internal space. Because $g_{i}=1$ at the sphere center $r=0$, we have $C_{3}=0$ and Equation (16) becomes

$$
g_{i}=\frac{1}{1-\alpha^{2} r^{2}}, \alpha^{2}=\frac{\chi}{3} \rho c^{2}=\frac{8 \pi \gamma}{3 c^{2}} \rho=\frac{4 \pi r_{g}}{3 m}
$$

in which Equations (7) and (14) are used to transform parameter $\alpha$.

\section{Boundary Conditions}

For the line element in Equation (1), the area of the surface $r=$ constant is $4 \pi r^{2}$, but the distance of this surface from the sphere center $r=0$ is not equal to $r$. For the internal space, we have using Equation (17)

$$
r_{i}=\int_{0}^{r} \sqrt{g_{i}} d r=\int_{0}^{r} \frac{d r}{\sqrt{1-\alpha^{2} r^{2}}}=\frac{1}{\alpha} \sin ^{-1}(\alpha r)
$$

For the external space, in accordance with Equation (15), we get

$$
\begin{gathered}
r_{e}=r_{i}(r=R)+\int_{R}^{r} \sqrt{g_{e}} d r=r_{i}(r=R)+\int_{R}^{r} \frac{d r}{\sqrt{1-r_{g} / r}} \\
=\frac{1}{\alpha} \sin ^{-1}(\alpha R)+\sqrt{r\left(r-r_{g}\right)}-\sqrt{R\left(R-r_{g}\right)}+\frac{r_{g}}{2} \ln \frac{r-\frac{r_{g}}{2}+\sqrt{r\left(r-r_{g}\right)}}{R-\frac{r_{g}}{2}+\sqrt{R\left(R-r_{g}\right)}}
\end{gathered}
$$

For $r=R$, Equations (18) and (19) yield $r_{i}=r_{e}$ which looks natural because the sphere outer surface is a physical object. The metric coefficients of the internal and the external spaces must be continuous at $r_{i}=r_{e}$ or at $r=R$, i.e., $g_{i}(R)=g_{e}(R)$. Using Equations (15) and (17), we have

$$
r_{g}=\alpha^{2} R^{3}=\frac{8 \pi \gamma \rho}{3 c^{2}} R^{3}
$$

Recall that $r_{g}$ is specified by Equation (14). Matching Equation (14) with Equation (20), we can find the sphere mass, i.e., 


$$
m=\frac{4}{3} \pi R^{3} \rho
$$

This is the mass of the sphere in Euclidean space. However, according to CTR, the space inside the sphere is semi-Riemannian, the metric coefficient $g_{i}$ is specified by Equation (17) and the sphere mass must be

$$
m=\rho \int_{0}^{2 \pi} d \theta \int_{0}^{2 \pi} \sin \phi d \phi \int_{0}^{R} r^{2} \sqrt{g_{i}} d r=\frac{2 \pi}{\bar{r}_{g}} \rho R^{3}\left(\frac{1}{\sqrt{\bar{r}_{g}}} \sin ^{-1} \sqrt{\bar{r}_{g}}-\sqrt{1-\bar{r}_{g}}\right)
$$

where

$$
\bar{r}_{g}=r_{g} / R
$$

To analyze Equation (22), decompose its right-hand part into the power series, i.e.,

$$
m=\frac{4}{3} \pi R^{3} \rho\left(1+\frac{3}{10} \bar{r}_{g}+\frac{9}{56} \bar{r}_{g}^{2}+\ldots\right)
$$

This result coincides with Equation (21) only for $\bar{r}_{g}=0$, i.e., only for Euclidean space. Recall that Equation (21) follows from the boundary condition for the metric tensor which must not be violated. Thus, for the line elements in Equation (1) the sphere mass corresponds to the Euclidean space though the metric coefficients of the space correspond to the Riemannian space. This allows us to suppose that in GTR these coefficients describe some other physical properties of space rather than the space geometry.

\section{Interpretation of Riemannian Space}

As known (Rashevsky, 1967), Riemannian space with $n_{R}$ dimensions can be embedded into Euclidean space with $n_{E}=n_{R}\left(n_{R}+1\right) / 2$ dimensions. For two-dimensional Riemannian space $\left(n_{R}=2\right)$, i.e. for an actual surface, we have $n_{E}=3$ which corresponds to the actual three-dimensional Euclidean space. However, for $n_{R}=3$, we get $n_{E}=6$, i.e. six-dimensional Euclidean space which has no physical interpretation. To demonstrate the idea of the proposed interpretation of the three dimensional Riemannian space (Vasiliev, 1989), consider a two-dimensional Euclidean space, i.e., a disk inside the circle with radius $r=R$ (Figure 1(a)) with area $\pi R^{2}$ and the metric coefficients $g_{r}^{0}=1, g_{\theta}^{0}=r^{2}$ and a surface of revolution in a three-dimensional Euclidean space (Figure 1(b)) with the metric coefficients $g_{r}=1+\left(z^{\prime}\right)^{2}, g_{\theta}=r^{2}$.

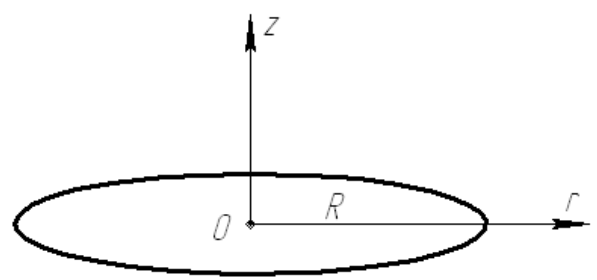

(a)

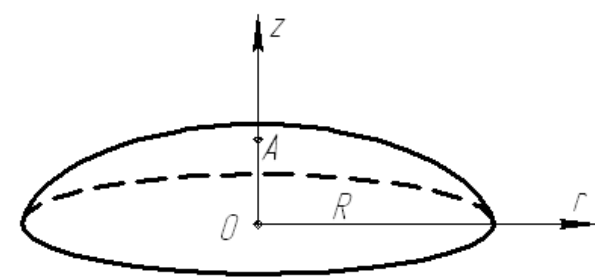

(b)

Figure 1. To the interpretation of Riemannian space

If we move point $\mathrm{A}$ in Figure 1(b) to the circle center $\mathrm{O}$ "compressing" the surface in the radial direction, the area of the resulting flat disk becomes equal to $\pi R^{2}$, but this disk exists now in non-homogeneous and anisotropic two-dimensional Euclidean space. Introducing the space "density" in the radial and the circumferential directions as

$$
\gamma_{r}=g_{r} / g_{r}^{0}=1+\left(z^{\prime}\right)^{2}, \gamma_{\theta}=g_{\theta} / g_{\theta}^{0}=1
$$

we can treat the Riemannian geometry as the mathematical model of non-homogeneous and anisotropic Euclidean space. Note that having Equations (9) and (10) which link the stress and the metric tensors, we can find the space "densities" as functions of stresses and, concentrating the material in the areas where the "densities" are high, arrive at some optimal material distribution corresponding to the acting stresses. Application of this approach to structural optimization is presented by Vasiliev and Fedorov (2006). 
In accordance with the foregoing interpretation of Riemannian space, gravitation causes not the space "curvature" which can be hardly imagined, but affects the "density" of the Euclidean space. The proposed interpretation does not change the physical results obtained in GTR. For example, the curvature of the light beam in the vicinity of a massive solid is induced in Newton theory by gravitational force, in GTR the beam follows the geodesic line of the space curved by the solid, whereas within the proposed interpretation, the beam propagates in space like in a transparent media with variable refraction coefficient.

\section{Singular Solutions}

As follows from Equations (15), the metric coefficient $g_{e}$ (or the "density") of the external space can become singular if the radial coordinate $r$ reaches the gravitational radius $r_{g}$. Hence, a natural question arises as to whether $r$ can be equal to $r_{g}$ in the external space. In the Euclidean space (see Section 6), the mass of the sphere with an arbitrary density $\rho(r)$ is

$$
m=4 \pi \int_{0}^{R} \rho(r) r^{2} d r
$$

Expressing $\rho$ from Equation (10) which is valid for any function $\rho(r)$, we get

$$
m=\frac{4 \pi}{\chi c^{2}} \int_{0}^{R}\left(r-\frac{r}{g_{i}}\right){ }^{\prime} d r=\frac{4 \pi R}{\chi c^{2}}\left(1-\frac{1}{g_{R}}\right)
$$

where $g_{R}=g_{e}(R)=g_{i}(R)$. Substituting this result in Equation (14) for $r_{g}$ and using Equation (7) for $\chi$, we arrive at the following expression for $r_{g}$ :

$$
r_{g}=R\left(1-\frac{1}{g_{R}}\right)
$$

Applying Equation (15) from which it follows that $g_{e}(R)=g_{R}$ is higher than unity, we can conclude from Equation (25) that $r_{g}<R$, i.e., that the surface with radius $r_{g}$ is located inside the sphere. However, Equation (15) for $g_{e}$ is valid outside the sphere and thus, cannot be singular. Note that Equation (25) is valid irrespective of the sphere structure and material.

As known, the singularity can appear at the sphere center $r=0$. Such singularity occurs for the pressure in the liquid sphere if $R=9 / 8 R$ (Weinberg, 1972) and in elastic sphere if $R$ approaches $r_{g}$ (Vasiliev \& Fedorov, 2012). Consider the orthotropic sphere for which no singularity can exist at the sphere center under some conditions imposed on the elastic properties of the sphere material.

\section{Classical Solution for an Orthotropic Sphere}

Within the framework of Newton gravitation theory, the set of equations describing the equilibrium of a spherically orthotropic solid under the action of gravitation forces includes the equilibrium equation (Love, 1927)

constitutive equations

$$
r \sigma_{r}^{\prime}-2\left(\sigma_{\theta}-\sigma_{r}\right)-k r^{2}=0, k=\frac{4 \pi}{3} \gamma \rho^{2}=\frac{\rho r_{g} c^{2}}{2 R^{3}}
$$

$$
\varepsilon_{r}=\frac{\sigma_{r}}{E_{r}}-\frac{2 v_{r \theta}}{E_{\theta}} \sigma_{\theta}, \varepsilon_{\theta}=\frac{1-v}{E_{\theta}} \sigma_{\theta}-\frac{v_{\theta r}}{E_{r}} \sigma_{r}
$$

and strain-displacement equations

$$
\varepsilon_{r}=u^{\prime}, \varepsilon_{\theta}=u / r
$$

in which $E_{r}$ is the radial elastic modulus and $E_{\theta}$ is the circumferential modulus of the material stiffness, $v$ are Poisson's ratios such that $v_{r \theta} E_{r}=v_{\theta r} E_{\theta}$, and $u(r)$ is the radial displacement. Eliminating $u$ from Equations (28), we arrive at the following compatibility equation which links the radial, $\varepsilon_{r}$, and the circumferential, $\varepsilon_{\theta}$, strains: 


$$
\left(r \varepsilon_{\theta}\right)^{\prime}=\varepsilon_{r}
$$

To solve the problem, satisfy the equilibrium equation, Equation (26), introducing the stress function $F$ as

$$
\sigma_{r}=\frac{F}{r^{2}}, \sigma_{\theta}=\frac{1}{2}\left(\frac{F^{\prime}}{r}-k r^{2}\right)
$$

Substituting the strains from Equations (27) in Equation (29) and expressing the stresses in terms of the stress function with the aid of Equations (30), we obtain the following governing equation for the stress function:

$$
r^{2} F^{\prime \prime}-m^{2} F=\frac{k r^{4}}{1-v}\left[3(1-v)+2 v_{r \theta}\right], m^{2}=\frac{2 E_{\theta}\left(1-v_{\theta r}\right)}{E_{r}(1-v)}
$$

The solution of this equation which satisfies the regularity condition at the sphere center $r=0$ is

$$
F(r)=C r^{n}+k B r^{4}, \quad n=\frac{1}{2}\left(1+\sqrt{1+4 m^{2}}\right), B=\frac{3(1-v)+2 v_{r \theta}}{\left(12-s^{2}\right)(1-v)}
$$

On the sphere surface, we have the boundary condition $\sigma_{r}(R)=0$. Determining the integration constant in Equation (32) from this condition, we arrive at the following final expressions for the stresses:

$$
\sigma_{r}=-k B R^{2}\left(\bar{r}^{n-2}-\bar{r}^{2}\right), \sigma_{\theta}=-\frac{1}{2} k R^{2}\left[B\left(n \bar{r}^{n-2}-4 \bar{r}^{2}\right)+\bar{r}^{2}\right]
$$

where

$$
\bar{r}=r / R
$$

The orthotropic properties of the sphere material are specified by parameters $m$ and $n$ in Equations (31) and (32). If the radial modulus $E_{r}$ is higher than the circumferential modulus $E_{\theta}$, i.e., the sphere is reinforced in the radial direction, we have $m^{2}<2, n<2$ and the stresses in Equations (33) become singular at the sphere center. If $E_{r}<E_{\theta}$, i.e., the sphere is reinforced with concentric shells, we have $m^{2}>2, n>2$ and the stresses in Equations (33) become zero at the sphere center. Thus, the classical solution shows that no singularity can appear at the center of an orthotropic sphere if $E_{r}<E_{\theta}$.

\section{GTR Solution for an Orthotropic Sphere}

The metric coefficient of the internal space is specified by Equations (17) and (20) which yield

$$
g_{i}=\frac{1}{1-r_{g} r^{2} / R^{3}}
$$

Substituting this result in Equation (9) and taking into account Equations (7), (14) and (21) for $\chi, r_{g}$ and $m$, we get

$$
\frac{h_{i}^{\prime}}{h_{i}}=\frac{r_{g} r}{R^{3}-r_{g} r^{2}}\left(1-\frac{3 \sigma_{r}}{\rho c^{2}}\right)
$$

The first governing equation of the problem under study follows from the conservation equation, Equation (8), if we substitute $h_{i}^{\prime} / h_{i}$ from Equation (36), i.e.,

$$
r \sigma_{r}^{\prime}-2\left(\sigma_{\theta}-\sigma_{r}\right)-\frac{\rho c^{2} r_{g} r^{2}}{2\left(R^{3}-r_{g} r^{2}\right)}\left(1-\frac{3 \sigma_{r}}{\rho c^{2}}\right)\left(1-\frac{\sigma_{r}}{\rho c^{2}}\right)=0
$$

Compare this equation with Equation (26) of the classical gravitation theory. For this purpose, perform linearization of Equation (37) neglecting the terms $\sigma_{r} / \rho c^{2}$ in comparison with unity. Then, Equation (37) reduces to

$$
r \sigma_{r}^{\prime}-2\left(\sigma_{\theta}-\sigma_{r}\right)-\frac{\rho c^{2} r_{g} r^{2}}{2\left(R^{3}-r_{g} r^{2}\right)}=0
$$

If we further neglect $r_{g} r^{2}$ in comparison with $R^{3}$, we arrive at Equation (26) of the classical gravitation theory. 
The last term in Equation (38) corresponds to the body force in Equation (26) and becomes infinitely high at the sphere surface $r=R$ if $R=r_{g}$. This property of the equation causes possible specific features of its solution discussed further in Section 10.

Return to Equation (37) which includes two unknown stresses $\sigma_{r}$ and $\sigma_{\theta}$. In the classical gravitation theory (Section 8), this equation is supplemented with the compatibility equation, Equation (29). To construct the analogous equation for GTR, apply the invariant condition of the Einstein tensor in Equations (4)-(6) ( Vasiliev \& Fedorov, 2006), assuming that this tensor identically satisfies the conservation equation, Equation (37), not only for the initial space, but for the deformed space as well. Introduce the metric tensor of the deformed space as

$$
g_{i}^{\varepsilon}=g_{i}\left(1+\varepsilon_{r}\right)^{2} \approx g_{i}\left(1+2 \varepsilon_{r}\right), \quad r_{\varepsilon}=r\left(1+\varepsilon_{\theta}\right)
$$

Here, index " $\varepsilon$ " corresponds to the deformed space and the strains $\varepsilon_{r}$ and $\varepsilon_{\theta}$ are assumed to be small in comparison with unity. As shown by Vasiliev \& Fedorov $(2006,2012)$, for the problem under study, the Einstein tensor satisfies the conservation equation for both initial and deformed states if $G_{I}^{l}\left(g_{i}^{\varepsilon}, r_{\varepsilon}\right)=G_{I}^{l}\left(g_{i}, r\right)$, here $G_{I}^{l}$ is specified by Equation (4). As a result, we arrive at the following equation:

$$
r \varepsilon_{\theta}^{\prime}\left(1+\frac{r h_{i}^{\prime}}{2 h_{t}}\right)+g_{i} \varepsilon_{\theta}=\varepsilon_{r}\left(1+\frac{r h_{i}^{\prime}}{h_{i}}\right)
$$

For $g_{i}=1$ and $h_{i}=1$ this equation reduces to Equation (29) of the theory of elasticity. Substituting $g_{i}$ and $h_{i}^{\prime} / h_{i}$ from Equations (35) and (36) in Equation (39), we get

$$
\left(r \varepsilon_{\theta}\right)^{\prime}-\varepsilon_{r}=\frac{r_{g} r^{2}}{R^{3}-r_{g} r^{2}}\left[\varepsilon_{r}-\varepsilon_{\theta}-\frac{r^{2}}{2} \varepsilon_{\theta}^{\prime}\left(1-\frac{3 \sigma_{r}}{\rho c^{2}}\right)-3 \varepsilon_{r} \frac{\sigma_{r}}{\rho c^{2}}\right]
$$

If we substitute the strains expressed in terms of stresses with the aid of Equations (27) in Equation (40), we can obtain the second equation which, being added to Equation (37), provides the set of two equations for the stresses $\sigma_{r}$ and $\sigma_{\theta}$. The final form of this set is

$$
\begin{gathered}
\bar{r} s^{\prime}-u=\phi(\bar{r})(1-3 s)(1-s)=0 \\
\bar{r} u^{\prime}+\left(1+2 \mu_{r \theta}\right) u+2 \bar{r}\left(1-\mu_{r \theta}\right) s^{\prime}-2\left(k \lambda_{\theta r}+2 \mu_{r \theta}-1\right) s \\
=2 \phi(\bar{r})\left\{2\left(k \lambda_{\theta r}+2 \mu_{r \theta}-1\right) s-\left(1+2 \mu_{r \theta}\right) u-\frac{\bar{r}}{2}(1-3 s)\left[u^{\prime}+2\left(1-\mu_{r \theta}\right) s^{\prime}-6 s\left(k \lambda_{r \theta} s+\mu_{r \theta} u\right)\right\}\right.
\end{gathered}
$$

where

$$
\begin{gathered}
s=\frac{\sigma_{r}}{\rho c^{2}}, \quad u=\frac{2}{\rho c^{2}}\left(\sigma_{\theta}-\sigma_{r}\right), \phi(\bar{r})=\frac{\bar{r}^{2} \bar{r}_{g}}{2\left(1-\bar{r}^{2} \bar{r}_{g}\right)} \\
k=\frac{E_{\theta}}{E_{r}}, \quad \mu_{r \theta}=\frac{v_{r \theta}}{1-v}, \quad \lambda_{r \theta}=\frac{1-2 v_{r \theta}}{1-v}, \lambda_{\theta r}=\frac{1-2 v_{\theta r}}{1-v}
\end{gathered}
$$

and $\bar{r}_{g}, \bar{r}$ are specified by Equations (23) and (34). The obtained equations must be integrated under the following boundary conditions: $\sigma_{r}(r=0)=\sigma_{\theta}(r=0)$ and $\sigma_{r}(r=R)=0$ which yield

$$
u(\bar{r}=0)=0, s(\bar{r}=1)=0
$$

As an example, consider an orthotropic sphere with zero Poisson's ratios, i.e., take $v=v_{r \theta}=v_{\theta r}=0$. Then, Equation (42) is simplified as

$$
\bar{r} u^{\prime}+u+2 \bar{r} s^{\prime}-2(k-1) s=2 \phi(\bar{r})\left\{2(k-1) s-u-\frac{\bar{r}}{2}(1-3 s)\left[u^{\prime}+2 s^{\prime}-6 k s^{2}\right)\right\}
$$

and includes only one material parameter $k=E_{\theta} / E_{r}$. Numerical (MAPLE-7) solutions of Equations (41) and (44) under boundary conditions in Equations (43) for $\bar{r}_{g}=0.1$ and three values of parameter 
$k=(0.9,1.0,1.5)$ are presented in Figure 2.

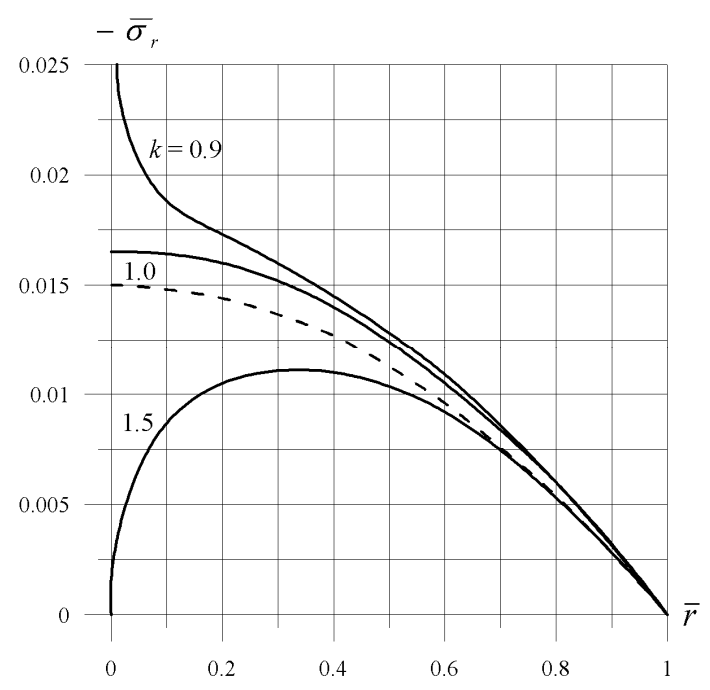

(a)

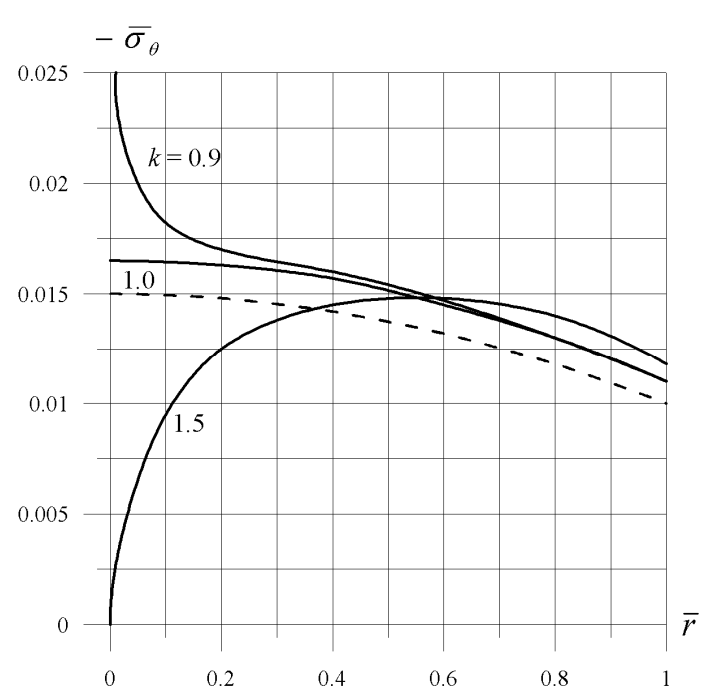

(b)

Figure 2. Dependences of the normalized radial (a) and circumferential (b) stresses $\bar{\sigma}=\sigma / \rho c^{2}$ on the radial coordinate for $\bar{r}_{g}=0.1$ and various values of parameter $k$

For comparison, the dashed lines demonstrate the classical solution in Equations (33) for an isotropic sphere with zero Poisson's ratio which takes the form

$$
\bar{\sigma}_{r}=-\frac{3 \bar{r}_{g}}{20}\left(1-\bar{r}^{2}\right), \quad \bar{\sigma}_{\theta}=-\frac{\bar{r}_{g}}{20}\left(3-\bar{r}^{2}\right)
$$

As can be seen, for $k=0.9$ the stresses are singular at the sphere center, for $k=1$ the stresses are finite and rather close to the classical solution, whereas for $k=1.5$, the stresses are zero at the sphere center. For the sphere whose radius $R$ is close to the gravitational radius $r_{g}$, i.e., for $\bar{r}_{g}=0.99$, the stresses are shown in Figure 3 for $k=1.5$ (solid lines) and for $k=1$ (dashed lines). Note that the classical solution in Equations (45) for the sphere with $\bar{r}_{g}=0.99$ gives the stresses that are by an order of magnitude lower than the stresses presented in Figure 3. For $\bar{r}_{g}=1$, the numerical procedure does not converge.

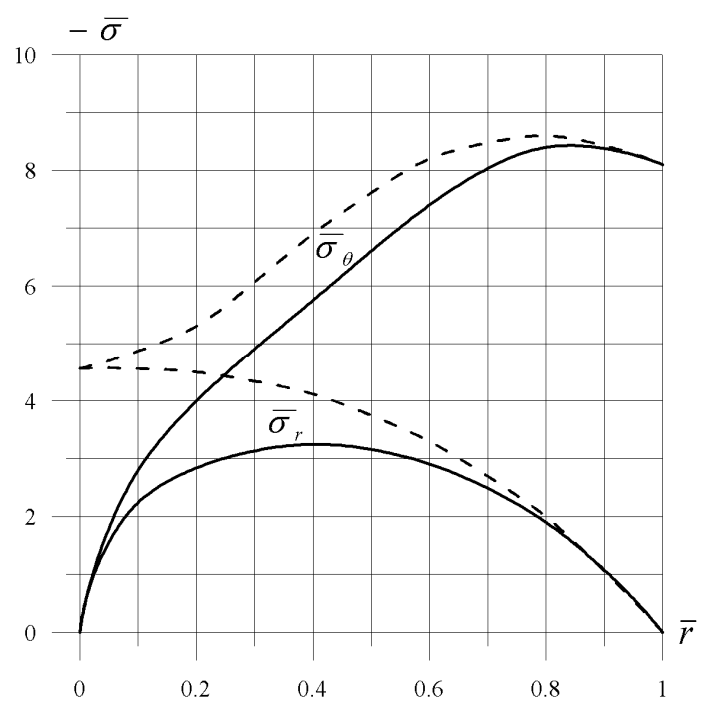

Figure 3. Dependences of the normalized stresses $\bar{\sigma}=\sigma / \rho c^{2}$ on the radial coordinate for $k=1.5 \quad(-)$ and for $k=1(--)$ and $\bar{r}_{g}=0.99$ 


\section{GTR Solution for an Orthotropic Sphere Covered with Liquid}

As follows from Figure 3, at the center of the orthotropic sphere with $k=1.5$ and $\bar{r}_{g}=0.99$ the stresses are zero. On the sphere surface $\bar{r}=1$, the radial stress is zero, whereas the circumferential stress is extremely high (recall that $\sigma=\rho c^{2} \bar{\sigma}$ ). Real materials cannot take such high stress and we can expect that while $R$ reduces to $r_{g}$, the surface layer of the sphere fails under increasing stress. To evaluate the conditions under which such failure occurs, we apply the stress intensity which for the problem under study has the following form (Jones, 2009):

$$
\sigma_{i}=\sigma_{\theta}-\sigma_{r}
$$

The material fails when $\sigma_{i}$ reaches some ultimate value established experimentally. Note that if $\sigma_{r}=\sigma_{\theta}$, and the failure does not occur. The condition $\sigma_{r}=\sigma_{\theta}=-p$ is satisfied for the liquid which does not fail under any pressure $p$. For a liquid sphere, the pressure can be found from Equations (37) which is simplified as

$$
\bar{p}^{\prime}+\frac{\bar{r}_{g} \bar{r}}{2\left(1-\bar{r}_{g} \bar{r}^{2}\right)}(1+3 \bar{p})(1+\bar{p})=0
$$

and has the following solution:

$$
\bar{p}(\bar{r})=-\frac{\sqrt{1-\bar{r}_{g} \bar{r}^{2}}-C}{\sqrt{1-\bar{r}_{g} \bar{r}^{2}}-3 C}
$$

in which $\bar{p}^{\prime}=p / \rho c^{2}$ and the integration constant $C$ can be found from the boundary condition on the sphere surface, i.e.,

$$
\bar{p}(\bar{r}=1)=0
$$

The resulting expression for the pressure is (Synge, 1960)

$$
\bar{p}(\bar{r})=-\frac{\sqrt{1-\bar{r}_{g} \bar{r}^{2}}-\sqrt{1-\bar{r}_{g}}}{\sqrt{1-\bar{r}_{g} \bar{r}^{2}}-3 \sqrt{1-\bar{r}_{g}}}
$$

The idea of the proposed solution is demonstrated in Figure 4 which corresponds to $\bar{r}_{g}=0.1$ and $k=1.5$.

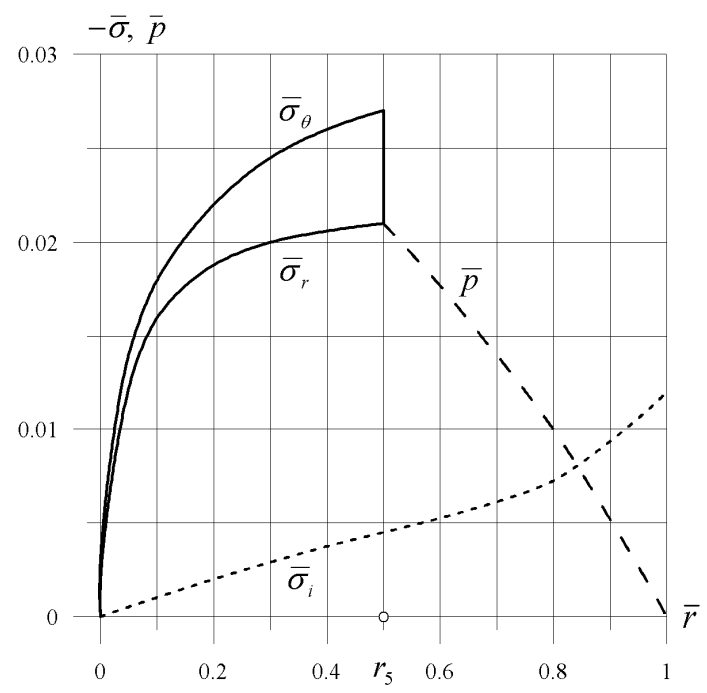

Figure 4. Dependences of the normalized stresses and pressure on the radial coordinate

\footnotetext{
- stresses in solid sphere

- - - pressure in liquid stress intensity in solid sphere
} 
Using the stresses presented in Figure 2, we can plot the stress intensity $\sigma_{i}$ in Equation (46) as a function of $\bar{r}$ (the dotted line in Figure 4). Assume that $\sigma_{i}$ reaches the ultimate value at some radius $\bar{r}=\bar{r}_{s} \quad\left(\bar{r}_{s}=0.5\right.$ in Figure 4). Then, the material of the external part of the sphere $\left(\bar{r}_{s} \leq \bar{r} \leq 1\right)$ can be simulated with liquid. The pressure in liquid is specified by Equation (50) which allows us to find the pressure $\bar{p}_{s}\left(\bar{r}=\bar{r}_{s}\right)$. The internal part of the sphere $\left(0 \leq \bar{r} \leq \bar{r}_{s}\right)$ is elastic and orthotropic. The stresses can be found by integration of Equations (41) and (44) under the following boundary conditions: $u(\bar{r}=0)=0$ and $s\left(\bar{r}=\bar{r}_{s}\right)=\bar{p}_{s}$.

Now return to the case $\bar{r}_{g}=1$. For the liquid sphere, the pressure specified by Equation (50) becomes infinitely high at the sphere center if the sphere radius $R$ is reduced to $9 / 8 r_{g}$ (Weinberg, 1972). However, for the laminated sphere under consideration, the central part is a solid orthotropic sphere with $k=1.5$ (Figure 3) and there is no singularity at the sphere center. For a solid orthotropic or isotropic sphere, the numerical solution gives high circumferential stress on the sphere surface $r=R$ and does not converge if $R=r_{g}$ which means that either the solution is singular at $r=R$ or that the solution does not exist for $R=r_{g}$. Unfortunately, the numerical solution does not allow us to identify which of these two cases takes place. However, for a sphere with a liquid surface layer, we have the analytical solution, Equation (48), which can be used to clear out the situation. Taking $\bar{r}_{g}=1$ and $\bar{r}=1$ in Equation (48), we get $\bar{p}=-1 / 3$ which has no physical meaning, first, because the pressure cannot be negative and, second, because the boundary condition in Equation (49) cannot be satisfied. Thus, the GTR problem has no solution for the sphere with radius which is equal to the gravitational radius. The reason for this is associated with Equation (38) in which the last term is analogous to the body gravitation force in Equation (26) of the classical gravitation theory. This force (or the space "density" in Equations (24)) becomes infinitely high on the sphere surface $r=R$ if $R=r_{g}$. It seems evident that in this case the boundary condition in Equation (49) cannot be satisfied, because the infinitely high force acting on the surface $r=R$ cannot be accompanied with zero pressure acting on this surface.

\section{Conclusion}

The foregoing results allow us to arrive at the following conclusions.

As follows from Equation (25) which links the gravitational radius and the value of the metric coefficient on the sphere surface and is valid for any model of the sphere material, the surface corresponding to the gravitational radius is always located inside the sphere. Thus, for the metric coefficient of the external space specified by Equation (15), $r$ cannot become equal to $r_{g}$ and the singularity of the metric coefficient in the external space does not exist irrespective of the structure and the material of the spherical solid.

The condition according to which the GTR solution for the external space must degenerate to the classical solution at a distance from the sphere surface actually means that the geometry of the space inside the sphere is Euclidean, and that Riemannian geometry of the inside space can be treated as a mathematical model of a non-homogeneous and anisotropic Euclidean space.

As shown in Sections 8 and 9, both classical and GTR solutions for the orthotropic sphere whose circumferential modulus is higher than the radial modulus, in contrast to the solutions for the incompressible liquid and the isotropic elastic solid spheres, do not demonstrate the singularity at the sphere center if the sphere radius reduces to the gravitational radius.

It is shown that for a specially constructed sphere which consists of an internal orthotropic elastic solid covered with incompressible liquid and whose external radius is equal to the gravitational radius, the GTR solution does not exist.

\section{References}

Feynman, R. P., Morinigo, F. B., \& Wagner, W. G. (1995). Feynman Lectures on Gravitation. Addison-Wesley Publising Company.

Fock, V. (1959). The Theory of Space, Time and Gravitation. London: Pergamon Press.

Hawking, S. W., \& Penrose, R. (1970). The singularities of gravitational collapse and cosmology. Proc. of the Royal Soc. A, 314, 529-548. http://dx.doi.org/10.1098/rspa.1970.0021

Jones, R. M. (2009). Deformation Theory of Plasticity. Blacksburg, Virginia: Bull Ridge Publishing.

Landau, L. D., \& Lifshitz, E. M. (1962). Field Theory. Moscow: Nauka (in Russian).

Logunov, A. A., \& Mestvirishvili, M. A. (1985). Foundations of Relativistic Gravitation Theory. Moscow State University (in Russian).

Schwarzschild, K. (1916a). Uber das Gravitationsfeld eines Massenpunkies nach der Einsteinschen Theorie. 
Sitzungsberichte der Deutschen Akademie der Wissenschaften zu Berlin, Klasse fur Mathematik, Physik, und Technik. 189.

Schwarzchild, K. (1916b). Uber das Gravitationfeld einer Kugel aus incompressibler Flussigkeit nach der Einsteinschen Theorie. Sitzungsberichte der Deutschen Akademie der Wissenschaften zu Berlin, Klasse fur Mathematik, Physik, und Technik. 424.

Synge, J. L. (1960). Relativity: the General Theory. Amsterdam: North Holland.

Vasiliev, V. V. (1989). Stressed state of solids and some geometrical effects. Mechanics of Solids, 5, 30-34.

Vasiliev, V. V., \& Fedorov, L.V. (2006). Geometric theory of elasticity and shape optimization of solids. Mechanics of Solids, 1, 16-27.

Vasiliev, V. V., \& Fedorov, L.V. (2012). On singular solutions in spherically symmetric ststic problem of general relativity. Applied Physics Research, 4(2), 166-174, http://dx.doi.org/10.5539/apr.v4n1p166

Weinberg, S. (1972). Gravitation and Cosmology. New York, NY: Willey. 